\title{
Rotation profile inversion in solar-like stars
}

\section{In the COROT framework}

\author{
J. Lochard ${ }^{1,2}$, R. Samadi ${ }^{2}$, and M. J. Goupil ${ }^{2}$ \\ 1 Institut d'Astrophysique Spatiale, Université Paris XI, 91405 Orsay Cedex, France \\ e-mail: jeremie. lochard@ias.u-psud.fr \\ 2 Observatoire de Paris, LESIA, CNRS UMR 8109, 92195 Meudon, France
}

Received 25 August 2004 / Accepted 3 February 2005

\begin{abstract}
The observation of a few mixed modes on solar-like oscillating stars would enable their rotation profile to be inverted with success. Here simulated data are used to show that it is possible to find models for solar-like stars that present stochastically excited mixed modes with detectable amplitudes. We take special care to build the mode set by computing the mode amplitudes and selecting those modes with amplitudes compatible with the performance of the forthcoming seismic space experiment, COROT. The frequency set is inverted for various cases where input and trial stellar models differ and where random noise is added to the splittings. We show it is possible to localize a rotation gradient and assess its magnitude. Moreover the use of inverse and forward procedures in parallel gives access to a large part of the profile. We provide several constraints to help the selection of such stars. One looks for a relatively evolved star (still on the main sequence) of $\simeq 1.5 M_{\odot}$ and the rotation rate on the surface should not be too small.
\end{abstract}

Key words. stars: oscillations - stars: rotation

\section{Introduction}

Helioseismology has proven to be a powerful tool for probing the interior of the Sun. In particular the inversion of the solar eigenfrequencies has provided localized information on the internal structure and rotation of our star (Gough et al. 1996; Basu \& Christensen-Dalsgaard 1997; Basu et al. 2000; Schou et al. 1998). Solar-like oscillations have now been detected for several other stars from the ground; $\alpha$ Cen A (Butler et al. 2004; Bedding et al. 2004), Procyon (Martić et al. 2004; Eggenberger et al. 2004), $\beta$ Hydri (Bedding et al. 2002), $\eta$ Bootis (Kjeldsen et al. 2003); for a review see (Bouchy \& Carrier 2003; Bedding $\&$ Kjeldsen 2003). Furthermore, ongoing and forthcoming space seismic missions (MOST, COROT) should provide much more accurate data.

The tools developed for helioseismology can be adapted to study stellar interiors; however, the transition from helioseismology to asteroseismology is not an easy task. Stars other than the Sun are not spatially resolved. Because of averaging over the stellar surface, only oscillation modes with low degrees will be detected. The available mode sets will be much more restricted than in the solar case. Several studies have nevertheless explored what we can expect from inversion to probe the structure of solar-like stars; e.g. Basu (2003) has reviewed the different attempts in this field using simulated data sets.

These attempts to invert solar-like oscillations focused mostly on inversion for the structure of the star (sound speed, density profile, etc.) It has been shown that structure inversion for solar-like stars is not as straightforward as for the Sun but remains possible. The success of the inversion is definitely linked to the number of observed modes bearing independent information, to the accuracy of the frequency measurement, but also to the quality of other observational constraints on the star, such as the basic stellar parameters which are usually poorly known. Rotational inversions based on simulated data sets have also been performed for $\delta$ Scuti stars, using opacity-driven modes (Goupil et al. 1996). It is expected that such inversions are unlikely to succeed with pure $p$ mode oscillations (see Christensen-Dalsgaard 2004). Gough \& Kosovichev (1993) showed successful rotational inversion results with solar-like oscillations; but the nature of the modes was not mentioned, and the relative error on the splittings was very small.

Obviously a restricted mode set and a degraded frequency resolution prevent the inversion process from providing comparable constraints on the stellar interiors to those on the solar interior. However we aim here at showing that for some specific stars, rotational inversion can be achieved using solar-like oscillations, primarily in the framework of the space seismic mission COROT. Indeed some stars must present a few mixed modes in the high frequency regime where modes are usually pure $p$ modes (see Christensen-Dalsgaard 2004). These mixed modes present dual characterisitics as they have kinetic energy both in the inner regions of the star ( $g$ mode nature) and in the 
outer layers ( $p$ mode nature). Such modes may have already been observed for the star $\eta$ Boo (Christensen-Dalsgaard et al. 1995; Guenther \& Demarque 1996; Di Mauro et al. 2003). We expect that observation of such modes might help to determine the variation of the rotation profile along the radius and localize some possible strong rotational gradient. This information would be valuable in providing useful constraints for the modelling of the angular momentum transport mechanisms inside the star.

To establish what one can expect from rotational inversion with low degree, high radial order modes, we needed to construct artificial "observational" datasets comprised of rotational splittings. To do so, we began selecting a stellar model and generating the frequencies and the rotational kernels associated with its eigenmodes; see Sect. 3. In this selection, our main criterion was the existence of a few mixed modes in the stochastically excited frequency range. As the mode set is crucial to the success of the inversion (Basu et al. 2002), we took special care in its selection (Sect. 4). We first formulated an a priori mode rejection process based on criteria such as the degree and the physical nature of the modes. Then we computed the oscillation amplitudes for the remaining modes according to our current knowledge of the excitation and damping processes. We also determined a detection threshold according to the expected performance of COROT. Amplitudes of the modes were then compared to the COROT threshold to select the set of modes (Sect. 4). Assuming a slow rotation with a given rotational profile, we then computed the rotational splittings which were optionally contaminated with random noise.

We then inverted the rotational splittings using the SOLA inversion technique (Sect. 2) for three different cases, from the ideal to a more realistic case: 1) inversion of the splittings using the input kernels; 2) inversion of the same splittings using trial kernels, i.e. kernels computed from a stellar model differing from the input model used to build the rotational splittings, where the trial model was selected according to observational constraints such as the location of the model in the HR diagram and the large and small frequency separations; 3 ) inversion with trial kernels and splittings contaminated by noise. The results of these inversions for rotation $-\Omega(r)$ - are presented in Sect. 5 .

\section{SOLA inversion technique}

For slow rotators, the rotational splitting of an $(\ell, n)$ mode $\left(\delta \omega_{n, \ell}\right)$ is related to the rotational rate along the radius, $\Omega(r)$, as follows:

$\delta \omega_{n, \ell}=\int_{0}^{1} k_{n, \ell}(r) \Omega(r) \mathrm{d} r+\epsilon_{n, \ell}$

where $k_{n, \ell}(r)$ is the rotational kernel and $\epsilon_{n, \ell}$ the error associated with the $(n, \ell)$ mode (Christensen-Dalsgaard 1998). Pure p mode rotational kernels are not highly localized, so that the splittings only provide an average of the rotation rate over their widths.

We use here the SOLA inversion method (Pijpers \& Thompson 1994), which aims at constructing an inversion kernel $K\left(r_{0}, r\right)$ localized in $r_{0}$ built as a linear combination of the rotational kernels, i.e.:

$K\left(r_{0}, r\right)=\sum_{j} c_{j}\left(r_{0}\right) k_{j}(r)$

where the subscript $j$ denotes the $(n, \ell)$ indices of the mode. $K\left(r_{0}, r\right)$ is sought to match a pre-selected target function $\mathcal{J}\left(r_{0}, r\right)$ as close by as possible, while keeping the effects of the data errors low. The aim is to minimize the following combination of the distance between $K$ and $\mathcal{J}$ and the data error propagation:

$\int_{0}^{1}\left(\mathcal{J}\left(r_{0}, r\right)-K\left(r_{0}, r\right)\right)^{2} \mathrm{~d} r+\mu \sum_{i j} \boldsymbol{E}_{i j} c_{i} c_{j}$,

where $\mu$ is a trade-off parameter to set the relative weight between the first and second terms in Eq. (3). $\boldsymbol{E}$ is the error variance-covariance matrix of the observed frequencies. Combining Eqs. (2) and (1) yields the average rotation rate around $r_{0}$ :

$\langle\Omega\rangle_{r_{0}} \equiv \sum_{j} c_{j} \delta \omega_{j}=\int_{0}^{1} K\left(r_{0}, r\right) \Omega(r) \mathrm{d} r+\sum_{j} c_{j}\left(r_{0}\right) \epsilon_{j}$.

The target function is chosen here as a Gaussian with width $\Delta$ :

$\partial\left(r_{0}, r\right)=\frac{1}{N} \exp \left(-\left(\frac{r-r_{0}}{\Delta}\right)^{2}\right)$

where $N$ is a normalization factor. The error on $\langle\Omega\rangle_{r_{0}}$ is $\Sigma_{j} c_{j} \epsilon_{j}$, with $\epsilon_{j}$ the errors on the observed splittings $\delta \omega_{j}$. For errors on the splittings independent of frequency $(\epsilon)$, the error magnification can be expressed as follows:

$\Lambda\left(r_{0}\right)=\left(\sum_{j}\left[c_{j}\left(r_{0}\right)\right]^{2}\right)^{1 / 2}$.

In this case, the error on $\langle\Omega\rangle_{r_{0}}$ is:

$\sigma_{\Omega\left(r_{0}\right)}=\Lambda\left(r_{0}\right) \epsilon$.

Hereafter rotation rates are normalized to the surface rotation rate $\Omega_{\text {surf }}$. Thus the errors are normalized as well.

\section{Stellar modelling}

Our stellar models were computed with the CESAM stellar evolution code (Morel 1997). This computation assumed the EFF equation of state, OPAL opacities (Iglesias \& Rogers 1996), the classical theory for convection (MLT), and the NACRE nuclear reaction rates (Gautier \& Morel 1997). Diffusion and rotation were neglected in the stellar model computations.

In the next sections the choice of the mass and the age of the stellar models is motivated by the expected amplitudes and the nature of the oscillation modes respectively. A correct choice of these parameters ensures that mixed modes exist in the stochastically excited frequency range. Numerical calculations showed that for main sequence stars with mass lower than $1.50 M_{\odot}$, mixed modes are less likely to appear in the appropriate frequency range. As a typical case, our input model had the 
following parameters: $M=1.55 M_{\odot}$, while the initial hydrogen and helium abundances were $X_{0}=0.705$ and $Y_{0}=0.275$. The mixing-length parameter was set to $\alpha=1.76$, and no overshoot was included for this model $\left(\alpha_{\mathrm{ov}}=0\right)$. Our input model was on the main sequence with a central hydrogen abundance $X_{\mathrm{c}}=0.10$. The input model and its evolutionary track are represented in Fig. 3. The input rotation profile, $\Omega(r)$, used in this study was built a posteriori, assuming local conservation of angular momentum.

\section{Selection of the mode set}

The ability to recover the correct rotation profile with an inversion process is strongly linked to the available mode set. To make this study as realistic as possible we selected the mode set on the basis of the expected amplitudes of the modes and of the constraints imposed by performance of the space seismic mission COROT (launch in 2006).

\subsection{A priori selection}

We selected a priori specific modes according to their nature and degree, $\ell$, since the quality of the inversion kernels depends on these properties. The depths which the modes probe depend very much on the nature of the modes. Pure $\mathrm{p}$ modes mainly probe the outer layers of the star where most of their kinetic energy is concentrated. Gravity modes - also named $g$ modes - have most of their energy in the deep interior. Their large inertia are responsible for their very small amplitudes on the surface. For this reason their detection seems unlikely (see Unno et al. 1989). However, for sufficiently massive stars the convective core recedes with evolution, which leads to an increase in the chemical composition gradient at the edge of the core. This gradient is directly involved in the expression of the Brunt-Väissälä frequency $N$. Figure 2 shows that $N^{2}$ increases at the edge of the core with the age of the star. For relatively evolved stars, modes with mixed nature appear. These mixed modes present a $g$ mode character in the deep interior and a $p$ mode character in the outer part of the star. They share their kinetic energy between these two regions, the degree of their "mixedness" depends on their fraction of energy in these regions. Some of these modes showing a dual nature have inertia that allows them to be detected, thus making it possible to probe the star at low radii.

We chose here to reject the pure gravity modes from our mode set and to keep only pure $p$ modes and those mixed modes with a rather low mixed character i.e. with their $p$ mode nature dominating over their $g$ mode nature.

The apparent amplitude of a mode is an average of the mode amplitude over the stellar surface, which strongly depends on the degree $\ell$ of the mode. The observable amplitude decreases with $\ell$. The forthcoming space missions, such as COROT, are expected to measure modes with $\ell \leq 3$. According to Libbrecht (1992) the signal to noise ratio and the uncertainty on the eigenfrequency determinations are linked; thus the frequencies of $\ell=3$ modes may be determined with a rather low precision. We therefore restrained ourselves to $\ell=1,2$ modes.

\subsection{Amplitude computation}

For these a priori selected modes, the root mean square amplitudes in terms of velocity, $v$ was calculated from the acoustic excitation rate $P$ and damping rate $\eta$, itself obtained from the tables of Houdek et al. (1999), calculated on the basis of Gough (1977a,b)'s non-local and time-dependent formulation of convection. We used the closest available model from the input model in Houdek's table of mass and temperature $\left(M=1.50 M_{\odot}\right.$ and $\left.T_{\text {eff }}=6400 \mathrm{~K}\right)$.

Excitation rates were computed according to the model of stochastic excitation by Samadi \& Goupil (2001). The calculations assumed a Lorentzian function for modelling the convective eddy time-correlations (see Samadi et al. 2003). For simplicity, we used the adiabatic assumption formulated by Kjeldsen \& Bedding (1995) to deduce the root mean square of the intrinsic mode amplitudes in terms of luminosity fluctuations $(\delta L / L)_{\text {intrinsic }}$ from their velocity $v$ according to:

$$
\left(\frac{\delta L}{L}\right)_{\text {intrinsic,max }}=\left(\frac{\delta L}{L}\right)_{\odot, \text { intrinsic,max }} \frac{V_{\max }}{V_{\max }^{\odot}} \sqrt{\frac{T_{\text {eff }, \odot}}{T_{\text {eff }}}} .
$$

One should point out that this relation has been established for bolometric amplitudes while COROT observes only in a finite bandwidth. Although this bandwidth is optimized for solar-like stars, the actual amplitudes are expected to be very similar to the bolometric ones. For the Sun we took the rms values $(\delta L / L)_{\odot, \max } \simeq 4 \mathrm{ppm}$ (see Kjeldsen \& Bedding 1995, Table 2; Barban et al. 2004) and $V_{\max }^{\odot} \simeq 27 \mathrm{~cm} / \mathrm{s}$ according to Chaplin et al. (1998)'s seismic observations. As the adiabatic assumption is not correct in the outer layers of a star, we performed a rough comparison with what is available in the literature (Houdek et al. 1999). At a high frequency, the adiabatic assumption underestimates the amplitudes compared to Houdek's results, so it is quite conservative in this case. At a low frequency a rough estimate showed that the adiabatic approach overestimates the amplitudes of the luminosity fluctuations; thus considering the non-adiabatic assumption would result in the present case losing one mode with a mixed character from the final mode set. This has no severe consequence for the inversion results. At the worst, it would mean that the present results are relevant for a star that is only a little more massive or evolved than our case.

Strictly speaking the theory underlying the excitation and damping rate computations used here is valid only for radial pure $p$ modes. Only non-radial modes present rotational splittings but the $\ell=1,2$ modes are quasi-radial in the zone where the excitation occurs. They are excited in the same manner as the radial modes. Then, as the excitation rate is proportional to the inverse of the mode inertia $I$, the excitation rates of the non-radial modes $P_{\ell}$ can be deduced from those of the radial ones $P_{0}$ according to the relation:

$\frac{P_{\ell}}{P_{0}} \simeq \frac{I_{0}}{I_{\ell}}$

for pure $p$ and low $\ell$ degree modes $I_{0} / I_{\ell} \simeq 1$. To some extent, it is the same for the damping rates. Indeed according to Balmforth (1992), the main contribution to damping is due to 


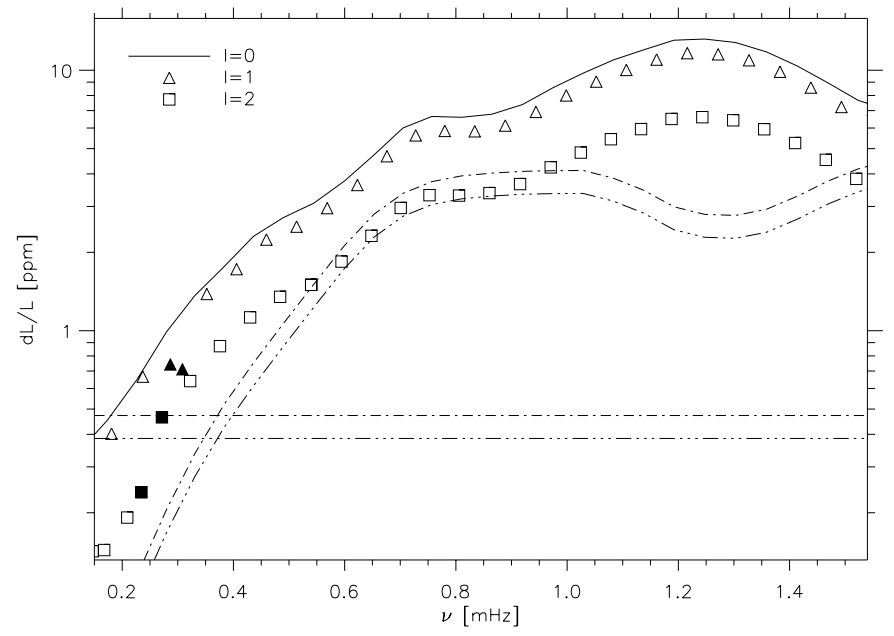

Fig. 1. Apparent mode amplitudes in terms of relative luminosity variations for $\ell=0,1,2$. The solid line shows the amplitude of the radial modes; the triangle and square symbols represent the $\ell=1$ and $\ell=2$ modes respectively. The filled symbols represent the mixed modes. The horizontal lines correspond to Eq. (15) and apply to modes whose widths are smaller than the frequency bin in the Fourier domain (i.e. modes with $v \lesssim 0.4 \mathrm{mHz}$ ). The upper line (dashed-dot) corresponds to $S N R_{\infty}=9$ (confidence level: 99\%) and the lower one (dashed-dot-dot-dot) to $S N R_{\infty}=6$ (confidence level: $95 \%$ ). The frequency-dependent thresholds apply to the other modes and correspond to Eq. (18) computed with $S N R=9$ (dashed-dot line) and $S N R=6$ (dashed-dot-dot-dot line).

convection and occurs mainly in the uppermost part of the convection zone where the modes are quasi-radial. Then we can deduce $\eta_{\ell}$ from $\eta_{0}$ using the relation:

$\frac{\eta_{\ell}}{\eta_{0}} \simeq \frac{I_{0}}{I_{\ell}}$

with $\eta$ being the damping rates. In the region where excitation and damping of the modes takes place, the mixed modes have the characteristics of pure $p$ modes. Thus Eqs. (9) and (10) also apply to mixed modes.

The intrinsic amplitude $\left(\frac{\delta L}{L}\right)_{\text {intrinsic }}$ and the apparent amplitude $\left(\frac{\delta L}{L}\right)$ of a mode with a degree $\ell$ are related by:

$\left(\frac{\delta L}{L}\right)_{\ell}=S_{\ell} *\left(\frac{\delta L}{L}\right)_{\text {intrinsic }, \ell}$

where $S_{l}$ is the spatial filtering function (see Christensen-Dalsgaard 1998). We used here the filtering functions used for the VIRGO instrument aboard SOHO spacecraft (Appourchaux et al. 1997), which observes the Sun as a star and monitors its luminosity variations as COROT and future asteroseismology space missions will do for other stars. $S_{l=1}$ and $S_{l=2}$ were set to 0.9 and 0.5 respectively, these coefficients depend on the angle between the rotation axis of the star and the line of sight. As an illustrative case we take the solar values. Figure 1 presents the apparent amplitudes of the luminosity variations for the $\ell=0,1,2$ modes. The mixed modes are found at low frequency, and their amplitudes clearly depart from those of the pure $p$ modes, while their smaller amplitudes are due to their larger inertia.

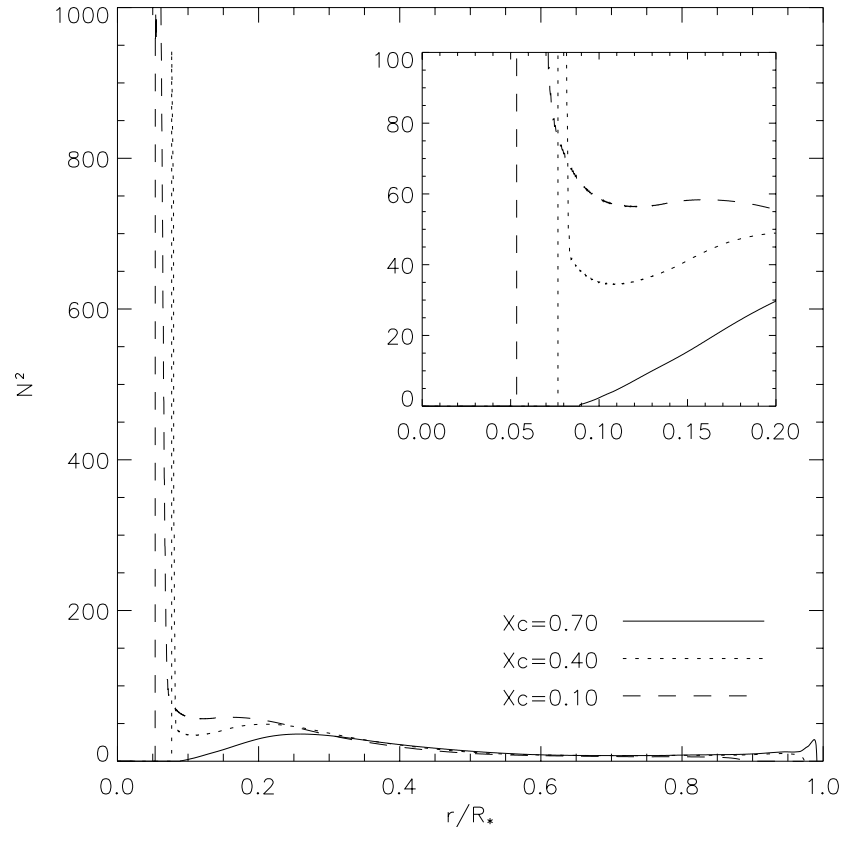

Fig. 2. Squared normalized Väissälä frequency for $1.55 M_{\odot}$ models at different evolutionary stages. The Väissälä frequency is normalized by $(G\langle\rho\rangle / \pi)^{1 / 2}$ with $\langle\rho\rangle$ the mean density and $G$ the gravitational constant. The solid line corresponds to the ZAMS model (the hydrogen abundance in the core is $X_{\mathrm{c}}=0.7$ ), the dotted line to an $X_{\mathrm{c}}=0.4$ model. The dashed line represents the Väissälä frequency for an $X_{\mathrm{c}}=0.10$ model. In the insert frame, we focus on the deep interior, where one can see that along evolution, at the edge of the core, a cavity where $g$ modes can be trapped develops in the high frequency regime.

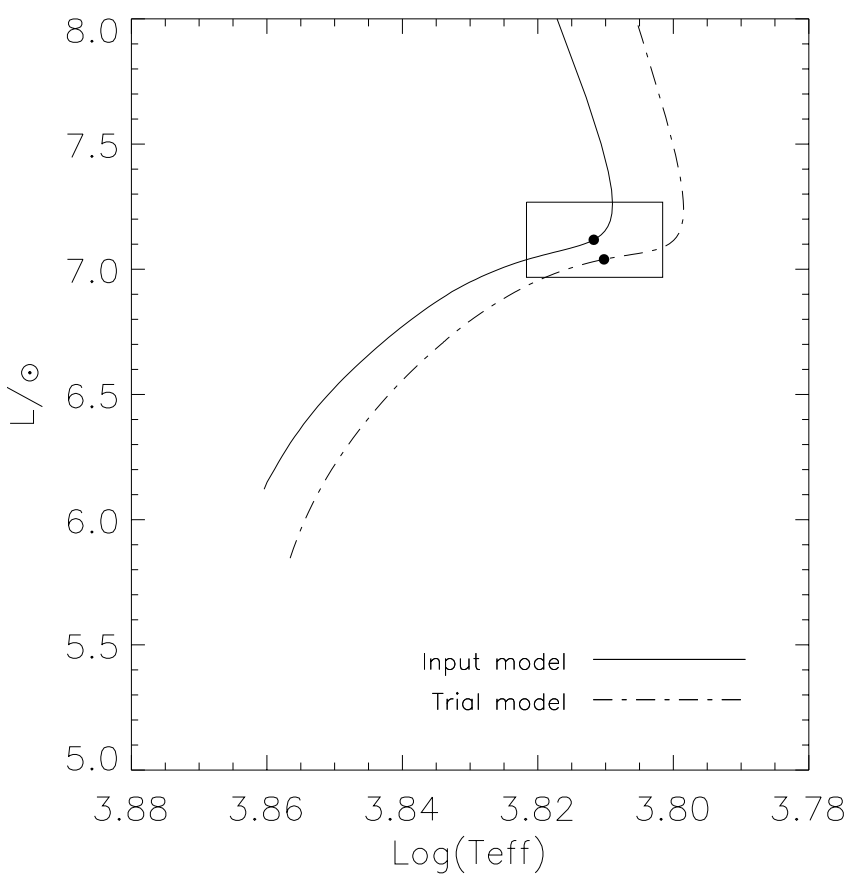

Fig. 3. Hertzsprung-Russell diagram with the evolutionary tracks of the input model (solid line) and the trial model (dashed line). Both models are represented by the black points on the tracks. The box materializes the typical uncertainties in determination of the luminosity and effective temperature. 


\subsection{COROT detection threshold and frequency accuracy}

Our detection threshold was based on COROT specifications. The white noise level over 5 days of observation will be $0.61 \mathrm{ppm}$ for a star of magnitude 5.7 (Auvergne \& COROT Team 2000); consequently the level of the photon noise in the power spectrum $B$ is (see Berthomieu et al. 2001):

$B=(0.61 \mathrm{ppm})^{2} 5$ days $=\frac{(0.61 \mathrm{ppm})^{2}}{2.3 \mu \mathrm{Hz}}(\mathrm{ppm})^{2} \mathrm{~s}$.

To set the threshold and to determine the precision at which a mode frequency can be measured, two distinct cases must be considered depending on the widths of the modes versus the frequency resolution of the spectrum (also refer to Berthomieu et al. 2001).

\subsubsection{Modes with long lifetimes}

Modes with lifetimes longer than the observation time have their widths in the frequency domain smaller than the frequency bin and hence correspond to a single peak in the power spectrum with height $H_{\infty}$ given by (see Berthomieu et al. 2001):

$H_{\infty}=\frac{T_{\mathrm{obs}}}{2}(\delta L / L)^{2}(\mathrm{ppm})^{2} \mathrm{~s}$,

where $\delta L / L$ is the root mean square of the observed apparent mode amplitude in intensity as defined in Eq. (11).

The corresponding signal to noise ratio ( $S N R$ hereafter) in the power spectrum, $S N R_{\infty}$, is then according to Eqs. (13) and (12):

$S N R_{\infty} \equiv \frac{H_{\infty}}{B}=\frac{1}{2} \frac{(\delta L / L)^{2}}{(0.61 \mathrm{ppm})^{2}} \frac{T_{\mathrm{obs}}}{5 \text { days }}$

where $T_{\mathrm{obs}}$ is expressed in unit of days. We stress that the $S N R$ is expressed in terms of power (i.e. in terms of the square of the mode amplitude) because theoretical evaluations of the mode amplitudes (see Sect. 4.2) are derived in terms of mean square. A possible alternative is to define a signal to noise in terms of amplitude as in Kjeldsen \& Frandsen (1992).

The value of $S N R_{\infty}$ does not affect the precision of frequency determination. Indeed, for long lifetimes, whatever the value of $S N R_{\infty}$, the uncertainty on the frequency determination corresponds to the size of the bin, that is, to $0.08 \mu \mathrm{Hz}$ for $T_{\text {obs }}=150$ days. However the value of $S N R_{\infty}$ sets the confidence level for the detection of an eigenmode. For $S N R_{\infty}=9$, the confidence level reached is $99 \%$.

For modes with long lifetimes, the detection threshold is from Eq. (14) then:

$$
\left(\frac{\delta L}{L}\right)_{\infty, \text { threshold }}=\sqrt{2 S N R_{\infty} \frac{5 \text { days }}{T_{\text {obs }}}} 0.61 \mathrm{ppm} .
$$

For $S N R_{\infty}=9$ (confidence level: $99 \%$ ), the threshold (Eq. (15)) is equal to $\simeq 0.47 \mathrm{ppm}$, and for $S N R_{\infty}=6$ (confidence level: $95 \%$ ) it is equal to $\simeq 0.39 \mathrm{ppm}$. These thresholds are shown in Fig. 1 by horizontal dashed lines and are relevant for modes whose widths are smaller than a single frequency bin, viz., modes below $v \simeq 0.4 \mathrm{mHz}$. Mixed modes are shown in Fig. 1 with filled symbols. Two $\ell=1$ modes lie above the $S N R_{\infty}=9$ threshold, while another $\ell=2$ stands between the $S N R_{\infty}=9$ and $S N R_{\infty}=6$ thresholds. The first two modes are detected with a $99 \%$ confidence level and the latter with a level above $95 \%$.

\subsubsection{Modes with short lifetimes}

For a mode with lifetime shorter than the observation time, the amplitude in the Fourier domain spreads over several frequency bins. In a first approximation the mode profile in the power spectrum is Lorentzian. The mean squared apparent amplitude of a mode is the integral over the peak profile. Consequently the height of the Lorentzian profile $H$ is linked to the mode apparent amplitude $\delta L / L$ and its width through the following relation (in $\mathrm{ppm}^{2} / \mu \mathrm{Hz}$ ) (see e.g. Baudin et al. 2005)

$H=\frac{(\delta L / L)^{2}}{\pi \Gamma}$

where $\Gamma=\eta / \pi$ is the mode line-width in $\mu \mathrm{Hz}$ with $\eta$ the damping rate. According to Eqs. (12) and (16) the signal to noise ratio $(S N R)$ then becomes:

$S N R \equiv \frac{H}{B}=\frac{(\delta L / L)^{2}}{(0.61 \mathrm{ppm})^{2}} \frac{2.3 \mu \mathrm{Hz}}{\pi \Gamma}$.

Again, for $S N R_{\infty}=9$, the mode is detected with a confidence level of $\sim 99 \%$. Thus from Eq. (17) the detection threshold for modes with short lifetimes is:

$\left(\frac{\delta L}{L}\right)_{\text {threshold }}=\sqrt{S N R \frac{\pi \Gamma}{2.3 \mu \mathrm{Hz}}} 0.61 \mathrm{ppm}$.

Hence, for $S N R=9$, a mode with amplitude $\delta L / L>$ $(\delta L / L)_{\text {threshold }}$ is detected with a confidence level better than $99 \%$. The threshold given by Eq. (18) is plotted in Fig. 1 for $S N R=9$ and $S N R=6$. Only modes with $S N R \geq 6$ were kept. Most of the modes were detected with a confidence level better than $99 \%$; however, few $\ell=2$ modes were detected with a confidence level between $99 \%$ and $95 \%$.

Note that the threshold depends only on $\Gamma$; as the width depends on the mode, the threshold also varies with frequency. We point out that Eqs. (16) and (12) assume that the Fourier transform is normalized with respect to $T_{\mathrm{obs}}^{-1 / 2}$, where $T_{\mathrm{obs}}$ is the duration of observation. This is why Eqs. (16) and (12) are independent of $T_{\mathrm{obs}}$. However whatever the choice of normalization, Eqs. (17) and (18) do not depend on $T_{\text {obs. }}$. Increasing the duration of observation does not increase the $S N R$ but does decrease the stochastic fluctuations of the mode profile leading to better precision when determining of the mode frequency. Indeed Eq. (2) in Libbrecht (1992) gives the precision at which the frequency of a mode - with a life time shorter than the observation time - can be measured for a given SNR. This precision depends on the observation time $\left(T_{\text {obs }}\right)$, on the mode line-width $(\Gamma)$, and also on the $S N R$ Eq. (17). Among the remaining modes with short lifetimes, the precision in frequency ranged between $\sim 0.05 \mu \mathrm{Hz}$ and $\sim 0.3 \mu \mathrm{Hz}$, according to Libbrecht (1992)'s formula. 


\subsection{Final set}

The selection above left us with a set of $50 \ell=1,2$ modes among which 3 were mixed modes. For these modes, we computed the associated rotational splittings according to Eq. (1) and assumed local conservation of angular momentum in the star. At high frequency, the widths of the modes increase. This might make the determination of the rotational splittings less accurate and some of the corresponding splitting useless; however, modes at such a frequency have very redundant kernels. The loss of their splittings does not affect the inversion results. In Sect. 5 below, we discuss the inversion results in cases of a set of splittings free of noise and one set contaminated with random noise. For short lifetime modes the error bars on the splittings were deduced from Eq. (2) in Libbrecht (1992). For modes with long lifetime, the error bars were set to the frequency bin.

\section{Results of inversion}

In this study we proceeded in 3 steps, successively aiming at more realistic conditions.

\subsection{Optimal case}

We first inverted the rotational splittings of our simulated data set, using the associated rotational kernels issued from the input model for $k_{j}$ in Eq. (2). These are the optimal conditions, to check how far we can recover the rotational profile. The modes become evanescent in the convective core for intermediate mass stars and it was not possible to recover the rotational information in that specific region. On the other hand, Fig. $6 \mathrm{a}$ shows that we properly retrieved the rotation rate from the edge of the core $\left(0.07 r / R_{*}\right)$ to $\simeq 0.30 r / R_{*}$ with $R_{*}$ the star radius. It was found that a few mixed modes only are sufficient to construct localized rotational kernels close to the core.

At intermediate radii, i.e. from $\simeq 0.30$ to $0.70 r / R_{*}$, it was not possible to construct localized inversion kernels. Neither pure $p$ modes nor mixed modes have enough energy at intermediate radii, as their rotational kernels do not peak in these regions.

Further up in the outer layers of the star, $p$ modes were sensitive to the rotation. The rotational kernels differ much more with the degree $\ell$ of the mode than with the mode frequency. As we assumed that we had access only to $\ell=1$ and 2 , the available $p$ mode rotational kernels were too redundant to enable to construct localized inversion kernels. To obtain an estimate of the rotation rate in these layers, we are left with the forward method. As in Soufi et al. (1998), one can write the $r$-dependent rotational profile $\Omega(r)$ as:

$\Omega(r)=\bar{\Omega}(1+\eta(r))$

where $\bar{\Omega}$ is the mean rotation rate. A rigid body rotation corresponds to $\eta(r)=0$. One can then write Eq. (1) as follows:

$\delta \omega_{n, \ell}=\bar{\Omega} \int_{0}^{1} k_{n, \ell}(r) \mathrm{d} r+\bar{\Omega} \int_{0}^{1} k_{n, \ell}(r) \eta(r) \mathrm{d} r$ and

$\int_{0}^{1} k_{n, \ell}(r) \mathrm{d} r=1-C_{n \ell}$

where $C_{n \ell}$ is the Ledoux constant in the inertial frame (see Unno et al. 1989, Eq. (19.46)). For pure $p$ modes, $C_{n \ell}$ can be neglected with regard to 1 . In the outer part of the star, rotation is expected to be almost rigid, i.e. $\eta(r) \simeq 0$. Thus the mean rotation rate in these layers can be expressed as:

$\bar{\Omega} \sim\left\langle\delta \omega_{n, \ell}\right\rangle$.

We selected a subset of rotational splittings of pure $p$ modes in the asymptotic frequency range ( 32 modes). We then computed the mean value of the subset. The extent of the pure $p$ mode kernels lead to a value which is an average over a large region. This mean value of the rotation rate is represented in Fig. 6a at $r / R_{*}=0.78$. The horizontal error bar extended between 0.55 to 1 stellar radius and was determined so that more than $95 \%$ of the kernel energy is located within this range for the subset of modes used for the forward computation. The vertical bar is the linear average of the errors on the splittings. One notices that the rotational rate obtained by forward computing is overestimated. This shift is linked to the relatively small extent of the rotational kernels in the deep interior where the rotational rate increases.

\subsection{Inversion with trial kernels}

The stellar model used in the inversion process is not expected to represent the real star exactly. This departure from reality also exists for the rotational kernels used for the inversion process. In our simulated experiment, therefore, we must assume that the rotational kernels issued from computed models would not match the real ones exactly. To study the impact of differences between the true and trial kernels, we inverted the splittings with rotational kernels associated with a stellar model differing from the input one. The input or reference model is the model described in Sect. 3. The trial model was determined as for a blind experiment, i.e. by trying to approach our reference model as close as possible.

We used two steps to constrain the trial model. The first step consisted in getting as close as possible to the input model using the HR diagram information. As for real observations, we drew an error box around the input model in the HR diagram with typical observational uncertainties $\left(\sigma_{T_{\text {eff }}}=150 \mathrm{~K}\right.$ and $\sigma_{L / L_{\odot}}=0.15$, Fig. 3). We built several models with various initial parameters and retained only those consistent with the error box. Then the asteroseismic constraints enabled us to make a selection among the remaining trial models. The comparison of the large and small separations of the trial and input models, as defined in Eqs. (23) and (24) below, gave additional constraints. The large separation - Eq. (23) - is mostly linked to the overall structure of the star and to its mean density. The small separation - Eq. (24) - is related to the chemical composition gradient close to the core, giving hints about the evolutionary stage of the reference model (see Christensen-Dalsgaard 2004).

$\Delta v_{n, \ell}=v_{n, \ell}-v_{n-1, \ell}$ 


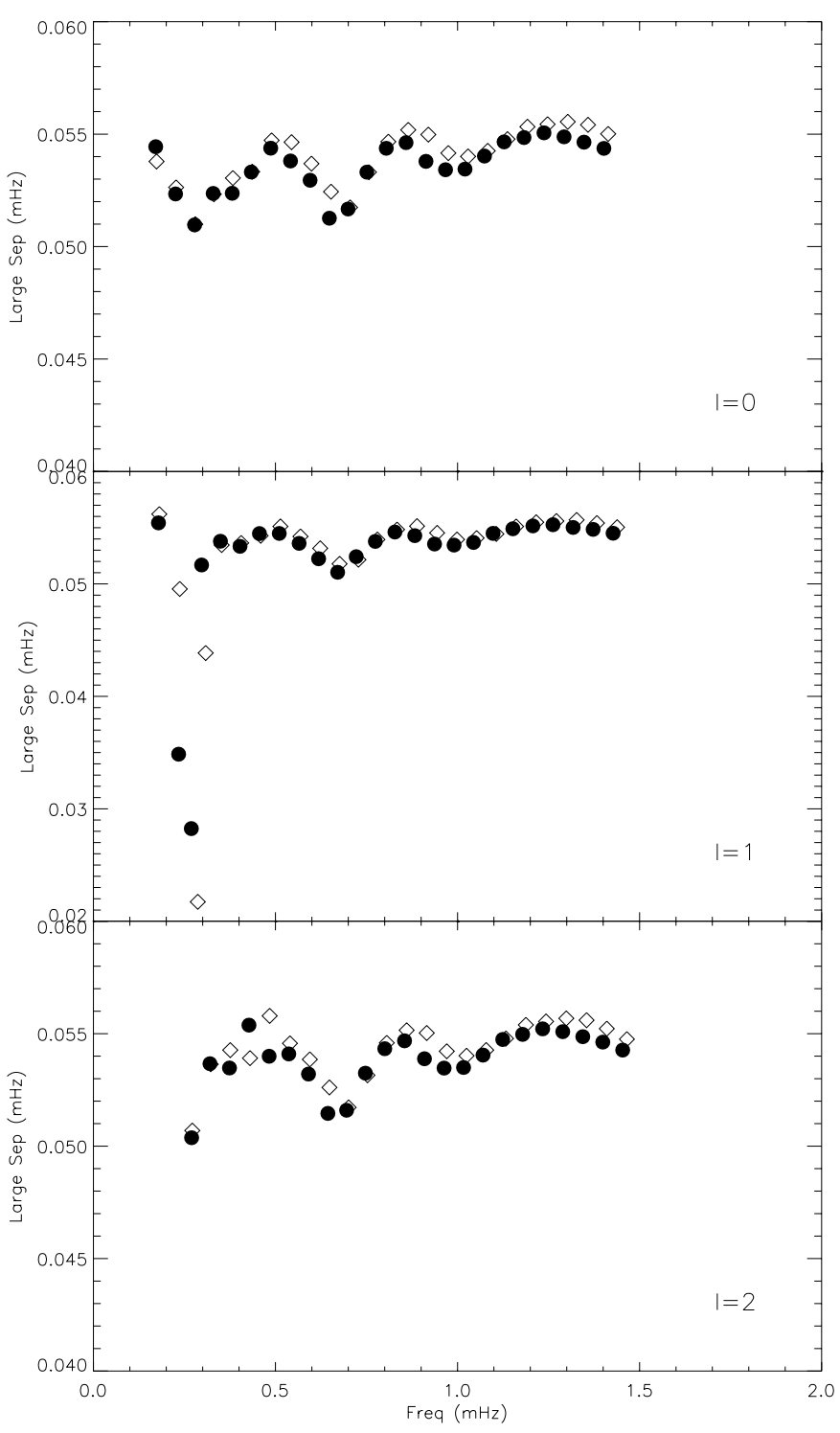

Fig. 4. Comparison of the large separations for the input and the trial models; see Eq. (23). Diamonds represent the large separation of the input model and filled circles correspond to that of the trial model. Each panel corresponds to a given degree $\ell$ (top panel: $\ell=0$ to bottom panel: $\ell=2)$.

$\delta v_{02}=v_{0, n}-v_{2, n-1}$.

For several models within the HR diagram error box, we could reproduce the large separation reasonably well (see Fig. 4). However, we could recover the small separation only over a part of the frequency range (see Fig. 5). We gave priority to the model reproducing the small separation in the interval $0.5-1 \mathrm{mHz}$, as we were mainly interested in low frequency modes; modes above $1 \mathrm{mHz}$ indeed produce very redundant rotational kernels. Eventually we selected the most appropriate model according to these criteria: $M=1.52 M_{\odot}, X_{0}=0.7$, $Y_{0}=0.28, \alpha=1.8$, and an overshoot parameter $\alpha_{\mathrm{ov}}=0.1$.

As was already the case when using the input kernels for the inversion, we recovered the rotational profile only partially. However, in this case the retrieved rates depart from the original profile, see Fig. 6b, although still in an acceptable way.

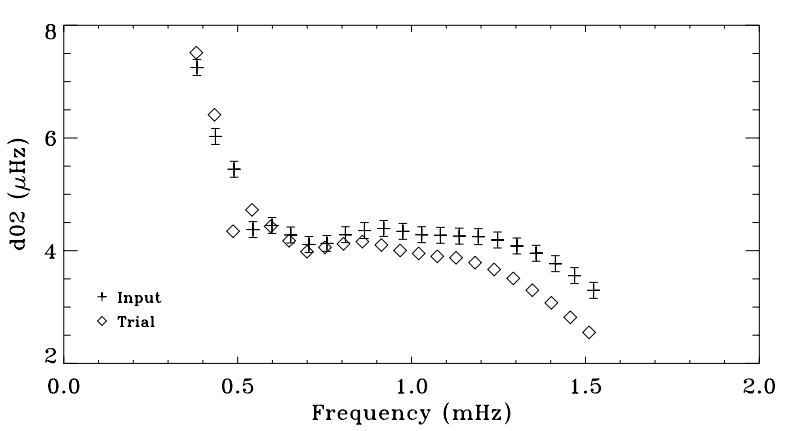

Fig. 5. Comparison of the small separations between $\ell=0,2-$ see Eq. (24) - for the input (crosses with associated error bars) and the trial models (diamonds).

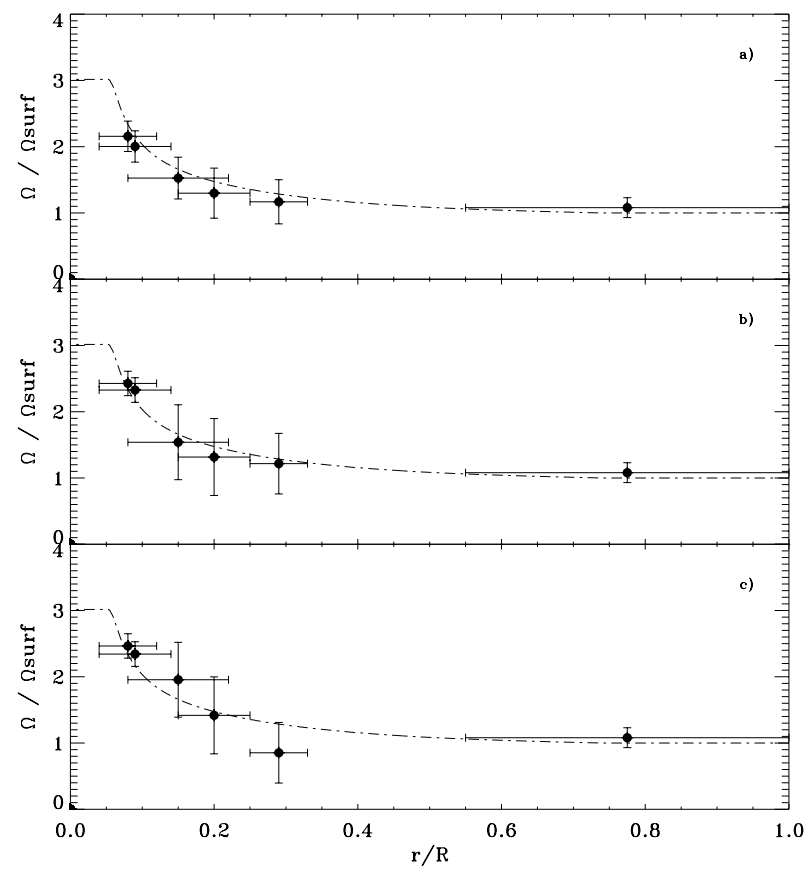

Fig. 6. Rotational profiles along the normalized radius of the stellar model. The dashed line represents the input rotation profile. The inverted rotation profiles are represented by black dots and their associated error bars: a) input rotational kernels, no noise included; b) trial rotational kernels, no noise included; c) one typical realization for trial rotational kernels, noise included $\left(V_{\text {surf }}=30 \mathrm{~km} \mathrm{~s}^{-1}, \epsilon=0.08 \mu \mathrm{Hz}\right)$. In each panel, the point at $0.75 r / R_{*}$ is derived from the forward computation (see Sect. 5.1). The vertical error bar represent a $1 \sigma$ error bars; the horizontal bars correspond to the width of the inversion kernels.

As previously, we used the forward computation to derive an estimate of the outer region rotation rate.

\subsection{Inversion with trial kernels and splittings including random errors}

Finally, the third case used the trial kernels and rotational splittings with random noise added prior to inversion. The noise was gaussian and several variance values were used corresponding to the COROT specifications and assumed surface rotation rates between 20 and $30 \mathrm{~km} \mathrm{~s}^{-1}$. The recovered rates for one typical realization are shown in Fig. 6c. The retrieved rates based on 


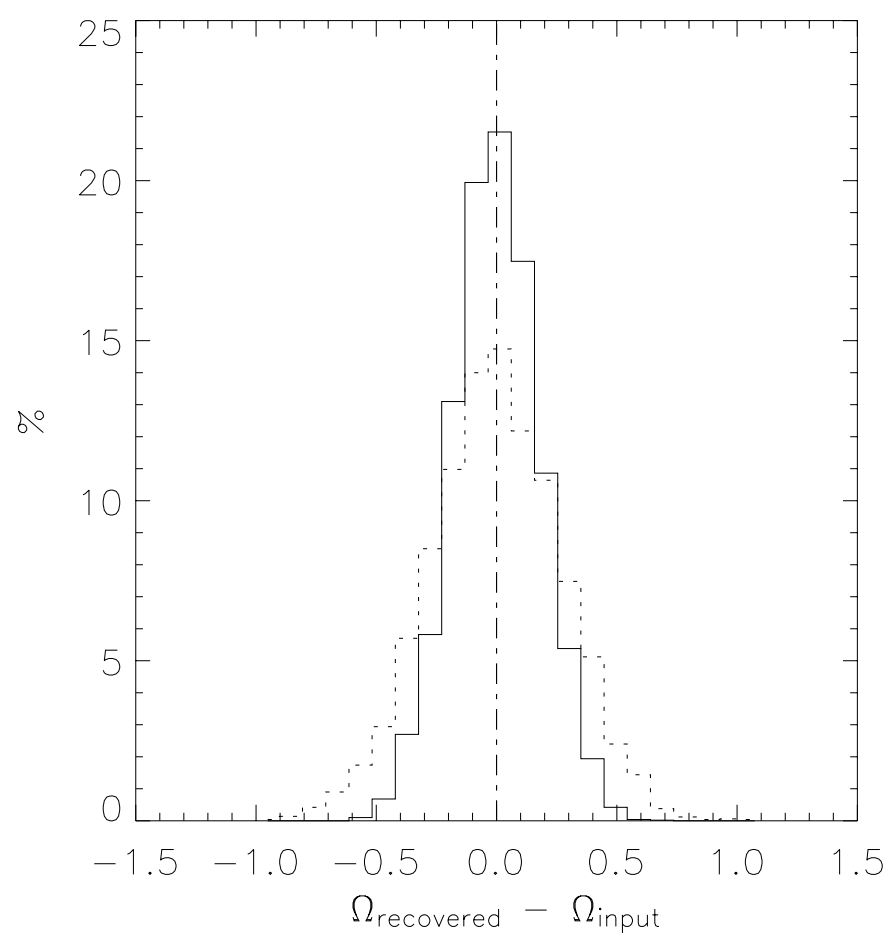

Fig. 7. Histogram of the differences between the recovered and input rotation rates at $r / R_{*}=0.08$, both rates were normalized to the surface rotation rate. The full and dashed lines correspond to $V_{\text {surface }}=30 \mathrm{~km} \mathrm{~s}^{-1}$ and $V_{\text {surface }}=20 \mathrm{~km} \mathrm{~s}^{-1}$, respectively. The vertical line corresponds to zero bias. The bins were set to $0.1 \Omega_{\text {surface }}$.

the set of splittings with random noise generally differ by a few percent from the retrieved rates based on noise-free splittings.

To test the efficiency of the inversion process in the presence of noise, we created 5000 realizations of noisy splittings and inverted them. In Fig. 7 we plotted the histogram of the difference between the recovered and the initial rates at a given radius for several uncertainty levels.

We first checked the validity of the error bars on the rotational rates retrieved by inversion shown in Fig. 6. These uncertainties, expressed in Eq. (7), correspond to magnification of the initial errors on the measured splittings through the inversion process. If unbiased they should match the dispersion of the 5000 recovered rates. We thus compared the standard deviation of these rates to the propagated errors and found that they matched extremely well at every radius. This shows that the errors bars are estimated without bias.

Secondly, we calculated the averages of the recovered rates at each radius over the 5000 realizations and compared them to the input rates. If there were no bias, mean value of the difference between the retrieved and the input rotation rates would be zero. There actually is a small nonzero difference at each inversion radius, which is identical to the departure between the input and the output rates when the inversion was processed without random noise. This bias is due to the finite widths of the inversion kernels, which set the ultimate limit in retrieving the input rotation rate.

In Fig. $6 \mathrm{c}$, the vertical error bars correspond to $1 \sigma$ normalized uncertainties on the rotation rate, which were computed

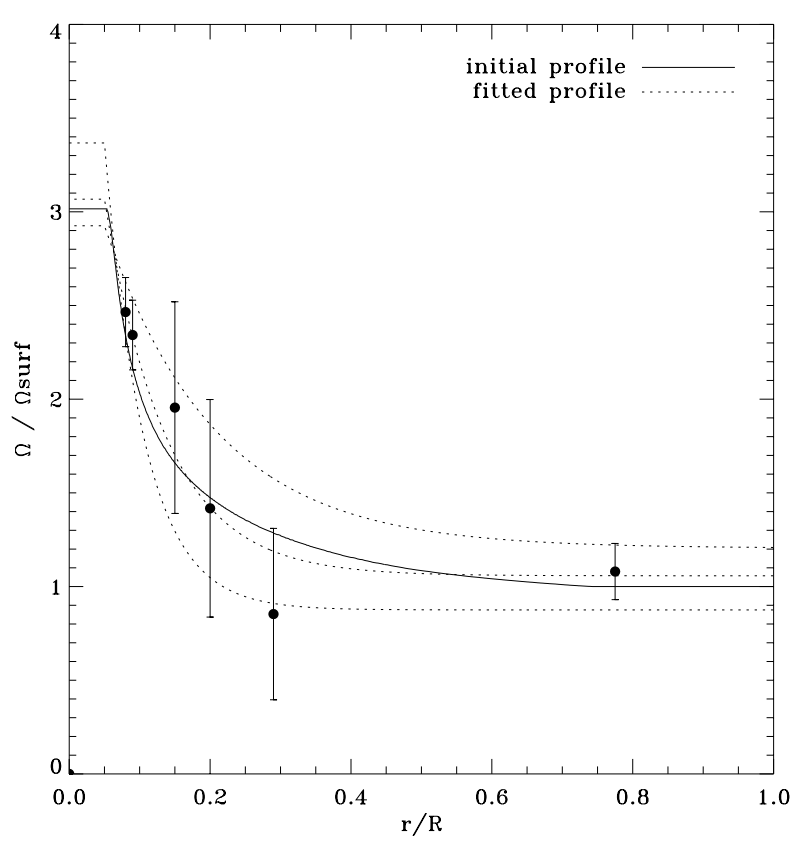

Fig. 8. Input and fitted rotational profiles. The full line curve stands for the initial rotational profile. The dotted curves fit the retrieved points of Fig. 6c. The middle one fits the central values, the two others fit the extreme values of the error bars.

assuming uncertainties on the splittings presented in Sect. 4.4 for a star rotating with a surface velocity of $V_{\text {surface }}=30 \mathrm{~km} \mathrm{~s}^{-1}$. This $\sigma$ value corresponds in Fig. 7 to the half-width of the distribution. In the best case -with full line $\left(V_{\text {surface }}=30 \mathrm{~km} \mathrm{~s}^{-1}\right)$ only a few percent of the random realizations in the tail of the gaussian distribution give misleading rotation rates. In the second case in Fig. 7 - dashed line - the $1 \sigma$ normalized error already corresponds to a large departure by the inverted rotation rate compared to the true value. In the conservative case, where we consider $3 \sigma$ error bars, a rotation gradient can still be measured - if large enough - in the best case in Fig. 7. This is probably no longer possible in the the worst case where the error bars strongly degrade our ability to estimate a rotational gradient.

As a final step, we estimated how discriminating the inversion is for reconstructing the rotational profile. We fitted the retrieved points with a decreasing exponential function. Figure 8 shows the mean and extreme profiles one could derive from the rates recovered in Fig. $6 \mathrm{c}$ where a random noise had been added to the splittings. We then computed the rotational splittings corresponding to these three profiles (mean and extreme). In Fig. 9 we plotted the input splittings - noise included - with black dots. The shaded area spans the values of the splittings computed from the extreme fitted profiles in Fig. 8. The input and the forward rotational splittings agree within the error bars.

This last step - i.e. computing the splittings from the recovered rotation rates - must be seen as a way to check the reliability of the inversion results. Inverted rotational profiles departing too much from the original will lead to computed splittings that do not match the true ones. 


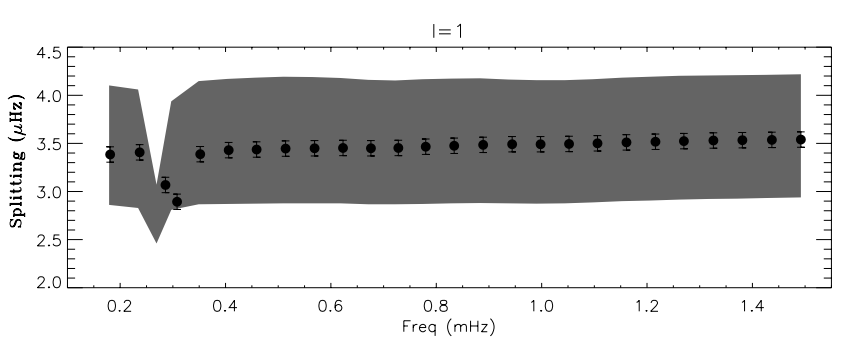

$1=2$

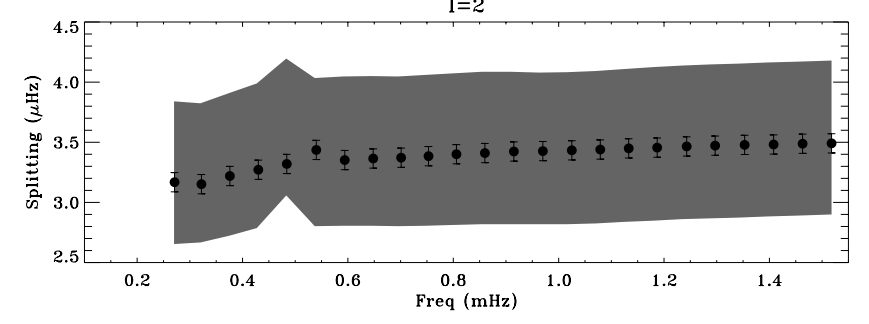

Fig. 9. Input and retrieved rotational splittings. The black dots represent the input splittings to which random noise was added, as in Fig. 6c. The shaded zone represents the range in which the solutions can be obtained by forward computation from the different fitted profiles in Fig. 8.

\section{Discussion}

As is well known, it is not possible to construct localized kernels for rotation with mode sets limited to $\ell=1,2$ and containing only pure $p$ modes. Introducing $\ell=3$ modes in the set improves the quality of the inversion kernels but does not provide enough diversity in the shapes of the rotational kernels to extract localized information at outer radii. However, we show here that stars exist which can present solar-like oscillations with a few mixed modes of detectable amplitudes. This presence in the mode set enables us to recover localized information on the rotation rate at several radii close to the core, leading to an estimate of the expected gradient.

As seen in Sect. 5.2, an important step is to model the observed star so as to build rotational kernels as close as possible to the real star. Combining the HR diagram and asteroseismic information (large and small separations) strongly constrains the model to be used to build the kernels. Accurate determination of stellar parameters, such as the luminosity and the effective temperature, is crucial at this step in the process.

We show that the inverse and forward procedures in stellar conditions are complementary and should be used in parallel. At low radii the inversion indeed provides localized and reliable information on the rotation rate and its evolution along the radius. In the outer part of the star the redundance of the pure $p$ mode rotational kernels does not allow us to build localized inversion kernels. Thus we derive the average rotation rate in this region directly from the splittings. Besides, the forward method can also be useful for checking the reliability of the rates retrieved by inversion. From the recovered points one can draw a rough rotational profile and use it to compute the splittings. By comparing the computed and the observed splittings it is possible to discard spurious profiles.

In short, a successful inversion of the rotational profile requires that the star fulfills several constraints. First, it has to be relatively evolved in order to have a receding convective core so that avoided crossing occurs in the proper frequency range (roughly for $X_{\text {core }} \leq 0.2$ ). Secondly, the mass range, which yields mixed modes with large enough amplitude to be detected, spreads from $\simeq 1.5 M_{\odot}$ up to masses that place the star in the instability strip of the HR-diagram, although studying stars above this upper mass limit is beyond the scope of this paper. For stars less massive than $\simeq 1.5 M_{\odot}$, it is unlikely that mixed modes are detectable within the stochastically excited frequency domain. One must see the present results as prospecting. Some assumptions such as the adiabatic one - see Sect. 4.2 - might be too optimistic, in this case mixed modes will be detected in solar-like oscillations for stars with slightly higher mass or at more evolved stages than the one presented in this paper.

Finally, the surface rotation rate of the "ideal" target star should not be too small so that the splittings can be determined with a good accuracy and that the relative errors on the splittings remain small enough. We must add that important physical effects have been neglected here. One will probably, for instance, have to consider the effects of rotation and of the magnetic field on the large and small frequency spacings (Dziembowski \& Goupil 1998). Moreover for faster rotators above $V_{\text {surf }} \simeq 20-30 \mathrm{~km} \mathrm{~s}^{-1}$ higher order effects on the rotational splittings will have to be considered.

Acknowledgements. The authors are grateful to Pr. Dziembowski for fruitful discussions and relevant comments. We thank $\mathrm{T}$. Appourchaux, F. Baudin, P. Boumier, E. Michel for useful remarks, and A. Mazumdar for improving the English. We also thank the anonymous referee for valuable comments and for his help in improving the manuscript.

\section{References}

Appourchaux, T., Andersen, B., Frohlich, C., et al. 1997, Sol. Phys., 170, 27

Auvergne, M., \& COROT Team. 2000, in The Third MONS Workshop: Science Preparation and Target Selection, 135

Balmforth, N. J. 1992, MNRAS, 255, 603

Barban, C., Hill, F., \& Kras, S. 2004, ApJ, 602, 516

Basu, S. 2003, Ap\&SS, 284, 153

Basu, S., \& Christensen-Dalsgaard, J. 1997, A\&A, 322, L5

Basu, S., Pinsonneault, M. H., \& Bahcall, J. N. 2000, ApJ, 529, 1084

Basu, S., Christensen-Dalsgaard, J., \& Thompson, M. J. 2002, in ESA SP-485: Stellar Structure and Habitable Planet Finding, 249

Baudin, F., Samadi, R., Goupil, M., et al. 2005, A\&A 433, 349

Bedding, T. R., \& Kjeldsen, H. 2003, PASA, 20, 203

Bedding, T. R., Kjeldsen, H., Baldry, I. K., et al. 2002, in ASP Conf. Ser., 259: IAU Coll., 185: Radial and Nonradial Pulsationsn as Probes of Stellar Physics, 464

Bedding, T. R., Kjeldsen, H., Butler, R. P., et al. 2004, ApJ, 614, 380 Berthomieu, G., Toutain, T., Gonczi, G., et al. 2001, in Proc. of the SOHO 10/GONG 2000 Workshop: Helio- and asteroseismology at the dawn of the millennium, 2-6 October 2000, Santa Cruz de Tenerife, Tenerife, Spain. ed. A. Wilson, Scientific coordination by P. L. Pallé (Noordwijk: ESA Publications Division), ESA SP-464, 411

Bouchy, F., \& Carrier, F. 2003, Ap\&SS, 284, 21

Butler, R. P., Bedding, T. R., Kjeldsen, H., et al. 2004, ApJ, 600, L75 
Chaplin, W. J., Elsworth, Y., Isaak, G. R., et al. 1998, MNRAS, 298, L7

Christensen-Dalsgaard, J. 1998, Lecture notes on stellar oscillations (Institut for Fysik og Astronomi, Aarhus Universitet)

Christensen-Dalsgaard, J. 2004, Sol. Phys., 220, 137

Christensen-Dalsgaard, J., Bedding, T. R., \& Kjeldsen, H. 1995, ApJ, 443, L29

Di Mauro, M. P., Christensen-Dalsgaard, J., Kjeldsen, H., Bedding, T. R., \& Paternò, L. 2003, A\&A, 404, 341

Dziembowski, W. A., \& Goupil, M.-J. 1998, in The First MONS Workshop: Science with a Small Space Telescope, held in Aarhus, Denmark, June 29-30, 1998, ed. H. Kjeldsen, \& T. R. Bedding, Aarhus Universitet, 69

Eggenberger, P., Carrier, F., Bouchy, F., \& Blecha, A. 2004, A\&A, 422, 247

Gautier, D., \& Morel, P. 1997, A\&A, 323, L9

Gough, D. 1977a, in Problems of Stellar Convection, IAU Coll., 38, 15

Gough, D. O. 1977b, ApJ, 214, 196

Gough, D. O., \& Kosovichev, A. G. 1993, in ASP Conf. Ser., 40: IAU Colloq. 137: Inside the Stars, 541

Gough, D. O., Kosovichev, A. G., Toomre, J., et al. 1996, Science, 272, 1296
Goupil, M.-J., Dziembowski, W. A., Goode, P. R., \& Michel, E. 1996, A\&A, 305, 487

Guenther, D. B., \& Demarque, P. 1996, ApJ, 456, 798

Houdek, G., Balmforth, N. J., Christensen-Dalsgaard, J., \& Gough, D. O. 1999, A\&A, 351, 582

Iglesias, C. A., \& Rogers, F. J. 1996, ApJ, 464, 943

Kjeldsen, H., \& Bedding, T. R. 1995, A\&A, 293, 87

Kjeldsen, H., Bedding, T. R., Baldry, I. K., et al. 2003, AJ, 126, 1483

Kjeldsen, H., \& Frandsen, S. 1992, PASP, 104, 413

Libbrecht, K. G. 1992, ApJ, 387, 712

Martić, M., Lebrun, J.-C., Appourchaux, T., \& Korzennik, S. G. 2004, A\&A, 418, 295

Morel, P. 1997, A\&AS, 124, 597

Pijpers, F. P., \& Thompson, M. J. 1994, A\&A, 281, 231

Samadi, R., \& Goupil, M.-J. 2001, A\&A, 370, 136

Samadi, R., Nordlund, Å., Stein, R. F., Goupil, M. J., \& Roxburgh, I. 2003, A\&A, 404, 1129

Schou, J., Antia, H. M., Basu, S., et al. 1998, ApJ, 505, 390

Soufi, F., Goupil, M. J., \& Dziembowski, W. A. 1998, A\&A, 334, 911

Unno, W., Osaki, Y., Ando, H., Saio, H., \& Shibahashi, H. 1989, Nonradial oscillations of stars, Nonradial oscillations of stars (Tokyo: University of Tokyo Press), 2nd ed. 